\title{
The Role of Neuromuscular Ultrasound when Diagnosing Chronic Inflammatory Demyelinating Polyneuropathy
}

\author{
Antonios Kerasnoudis, MD
}

Neurologist, Department of Neurology, St Josef-Hospital, Ruhr University, Germany

\begin{abstract}
Chronic inflammatory demyelinating polyneuropathy (CIDP) is the most common acquired immune-mediated inflammatory disorder of the peripheral nervous system. The diagnosis is based in classic cases, on the distribution pattern of the neurologic semiology, and pathologic changes of nerve conduction studies (NCS). However, in cases with subtle clinical presentation, an extended diagnostic workup may be needed (cerebrospinal fluid examination, laboratory tests, nerve biopsy). NCS remain fundamental for the diagnosis, follow up, and measurement of response to immune-treatment in CIDP. However, new challenges arose on how best to acquire a static and dynamic imaging of the peripheral nerves, with the aim of providing a holistic approach to the nerve impairment. According to the literature, neuromuscular ultrasound is able to detect in cases of CIDP thickened or swollen roots, peripheral nerves, or brachial plexus, findings that are consistent with ongoing inflammation. This review provides a timely update on the nerve ultrasound findings of CIDP and future possibilities of neuromuscular ultrasound are also discussed.
\end{abstract}

\section{Keywords}

Chronic inflammatory demyelinating polyneuropathy, nerve ultrasound, nerve hypertrophy, conduction block, immune-mediated neuropathies, brachial plexus

Disclosure: The author has no conflicts on interest to declare.

Received: February 9, 2013 Accepted: March 21, 2013 Citation: US Neurology, 2013;9(1):52-4 DOI: 10.17925/USN.2013.09.01.52

Correspondence: Antonios Kerasnoudis, MD, Department of Neurology, St Josef-Hospital, Ruhr University, Gudrunstr 56, 44791 Bochum, Germany.

E: antonis.kerasnoudis@gmail.com

Chronic inflammatory demyelinating polyneuropathy (CIDP) is an acquired immune-mediated inflammatory disorder of the peripheral nervous system with an estimated prevalence of about 0.5 per 100,000 children and one to two per 100,000 adults. 1,2 Typical CIDP arises between 30 and 60 years of age and is characterized by progressive, symmetric, proximal, and distal muscle weakness, paresthesias, sensory dysfunction, impaired balance, and reduced or diminished tendon reflexes, which evolve slowly over at least 8 weeks. The course can be either monophasic with stepwise progression or relapsing with spontaneous remissions. ${ }^{3}$ The diagnosis is based, in typical cases, on the time course, distribution pattern of nerve impairment, and results of the nerve conduction studies (NCS), which are frequently consistent with a motor and sensory demyelinating polyradiculoneuropathy, with evidence of conduction block and temporal dispersion. 4,5 These findings may be further supported with evidence of cytoalbuminologic dissociation in cerebrospinal fluid analysis, but this is not obligatory for the diagnosis. ${ }^{6}$ Different criteria, such as Inflammatory Neuropathy Cause and Treatment (INCAT), American Academy of Neurology (AAN), and Saperstein, can be used to define the disease. ${ }^{7,8}$

While nowadays NCS remain fundamental to confirm the presence, pattern, and severity of this type of polyradiculoneuropathy, ${ }^{6}$ new challenges have arisen recently in terms of how to acquire the best static and dynamic imaging of the relevant nerve structures in CIDP, with the aim of providing a complementary and holistic approach to nerve impairment. Using magnetic resonance imaging (MRI), various hypertrophic changes have been demonstrated in peripheral nerves, roots, or brachial plexus in studies on CIDP patients. ${ }^{9-14}$ Although MRI is an accurate diagnostic method in imaging soft tissues, it has the disadvantages of being expensive, timeconsuming, affected by artefacts (e.g. metal), and not practical, especially when a number of nerves need to be examined over a long course in patients with polyneuropathy. The role of neuromuscular ultrasound in the diagnostic workup of CIDP and polyneuropathies, in general, remains less well defined and parallels the beginnings of research of entrapment neuropathies. Only a few studies in the literature have used ultrasound to examine the pathologic changes in immune-mediated neuropathies, highlighting mainly pathologic changes of the cross-sectional area (CSA) of peripheral nerves and their correlation with clinical and electrophysiologic findings. ${ }^{15-21}$ This review provides an update on the diagnostic role of neuromuscular ultrasound in the diagnostic of CIDP, while possible future possibilities of neuromuscular ultrasound are also discussed.

\section{Quantification of Ultrasound Findings in Immune-mediated Neuropathies}

CSA reference values for peripheral nerves and brachial plexus have been reported in various studies in the literature. ${ }^{22-27}$ The difficulty, however, to differentiate a normal from a pathologic heterogeneity of CSA changes in 
peripheral nerves, especially in CIDP cases, remains an important limitation of neuromuscular ultrasound in clinical practice. Novel ultrasound measures, aiming to quantify pathologic ultrasound changes of peripheral nerves in immune-mediated polyneuropathies, have been recently introduced in the literature: (1) the intranerve CSA variability (for each nerve), defined as maximal CSA/minimal $\mathrm{CSA}^{28}$ (2) the internerve CSA variability (for each patient), defined as nerve with maximal intranerve CSA variability/nerve with minimal intranerve CSA variability, ${ }^{28}$ (3) the side-to-side difference ratio of the intranerve CSA variability (SSDIVA) (for each nerve), defined as side with maximal intranerve CSA variability/side with minimal intranerve CSA variability, ${ }^{29}$ and (4) the intraplexus CSA variability defined as: maximal CSA of the brachial plexus/minimal CSA of the brachial plexus (see Table 1). ${ }^{29}$

Using the intranerve CSA variability, the sonographer may differentiate in immune-mediated neuropathies focal (higher values) from diffuse (lower values) nerve enlargement, while the internerve CSA variability may reveal possible distribution patterns of peripheral nerve impairment. ${ }^{28,29}$ On the other hand, the side-to-side difference ratio of the intranerve CSA variability may be useful in detecting any lateralization of pathologic changes and the intraplexus CSA variability in differentiating focal (higher values) from diffuse (lower values) brachial plexus enlargement. ${ }^{29}$

\section{Ultrasound Findings}

Currently 10 studies (evaluating a total of 74 cases) on nerve sonography in CIDP patients have been published (see Table 2). The first description of the sonographic findings in CIDP was published from Taniguchi et al. ${ }^{30}$ In this report, the authors documented a brachial plexus hypertrophy on both sides and peripheral nerve hypertrophy at several sites of the median, sciatic and femoral nerve. Similar findings had only been reported in MRI studies until then. ${ }^{9-14} \mathrm{~A}$ possible explanation of the documented hypertrophy (see Figure 1) could derive from the classic 'onion-bulb' histologic appearance of the nerves in CIDP, as a result of recurrent episodes of demyelination and remyelination. ${ }^{31}$

After this initial publication, several years passed until the first systematic ultrasound study of CIDP patients was published. In 2004, Matsuoka et al. reported the ultrasound findings of the cervical roots in 13 patients with CIDP and 35 healthy individuals. ${ }^{32}$ The authors demonstrated a hypertrophy of the cervical roots in nine out of 13 patients with CIDP, a finding that seemed to correlate with the elevated levels of protein in cerebrospinal fluid. Another ultrasound study of 36 CIDP patients from Zaidman et al. confirmed the presence of diffuse nerve enlargements in peripheral nerves in this type of immune-mediated nerve injury. ${ }^{15}$ Similar findings have been reported in several case reports in the following years. ${ }^{33-36}$ These findings showed a correlation with the disease duration and nerve conduction findings in a small group of CIDP patients. ${ }^{37}$ Conversely, Rajabally et al. compared the distal median nerve CSA of 14 CIDP patients to 14 patients with sensory axonal neuropathy of various etiologies (including alcoholism, vitamin deficiency, impaired glucose tolerance, vasculitis, idiopathic). The authors concluded that the CSA of the median nerve was greater in CIDP compared with other polyneuropathies (sensitivity $57 \%$; specificity of $93 \%$ ). ${ }^{38}$

Another important aspect in the field of sonography in CIDP is the possible use of this method for identifying nerve conduction blocks. The localization of the nerve conduction block is often difficult to be made in the nerve
Table 1: Equations for Calculating the Intranerve-, Internerve-, Intraplexus Cross-sectional Area Variability and Side-toSide Difference Ratio of the Intranerve Cross-sectional Area Variability

\begin{tabular}{|c|c|c|}
\hline $\begin{array}{l}\text { Intranerve CSA variability } \\
\text { (for each nerve) }\end{array}$ & $=$ & $\begin{array}{l}\text { Maximal CSA } \\
\text { Minimal CSA }\end{array}$ \\
\hline $\begin{array}{l}\text { Internerve CSA variability } \\
\text { (for each subject) }\end{array}$ & $=$ & $\begin{array}{l}\text { Peripheral nerve with maximal } \\
\text { intranerve CSA variability } \\
\text { Peripheral nerve with the minimal } \\
\text { intranerve CSA variability }\end{array}$ \\
\hline $\begin{array}{l}\text { Side-to-side difference ratio } \\
\text { of the intranerve CSA } \\
\text { variability (for each nerve) }\end{array}$ & $=$ & $\begin{array}{l}\text { Side with the maximal intranerve } \\
\text { CSA variability } \\
\text { Side with the minimal CSA } \\
\text { intranerve CSA variability }\end{array}$ \\
\hline $\begin{array}{l}\text { Intraplexus CSA variability } \\
\text { (for each brachial plexus) }\end{array}$ & $=$ & $\begin{array}{l}\text { Maximal CSA of brachial plexus } \\
\text { Minimal CSA of brachial plexus }\end{array}$ \\
\hline
\end{tabular}

CSA $=$ cross-sectional area.

Figure 1: Longitudinal (A) and Axial (B) Sonographic Scan of the Median Nerve of a Chronic Inflammatory Demyelinating Polyneuropathy Patient

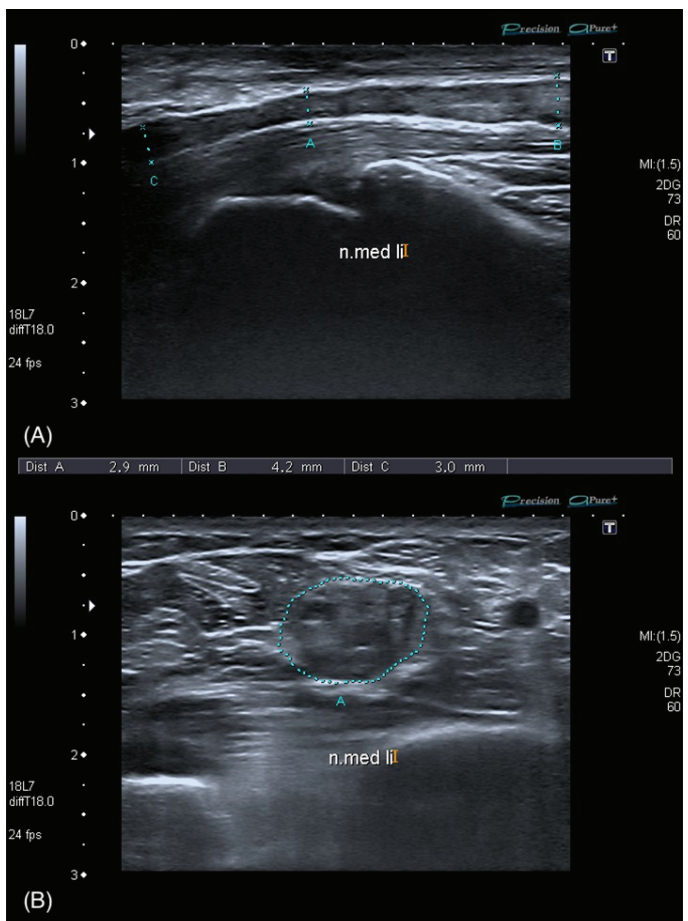

Longitudinal (A) and axial (B) sonographic scan of the left median nerve of a chronic inflammatory demyelinating polyneuropathy (CIDP) patient during clinical deterioration, showing an almost completely hypoechoic and edematous nerve, with loss of fascicular echostructure and enlargement of the cross-sectional area.

conductions studies, especially when dealing with proximal parts of the nerves. By overlooking this typical electrophysiologic finding of this disease, a delay in the diagnosis and therefore treatment can occur. In three CIDP cases in the literature, a correlation between the site of hypertrophy detected with ultrasound and the site of conduction block detected with NCS was demonstrated. ${ }^{17,33,39}$ Although this seemed to be a promising development, it is worth noting that Zaidman et al. failed to confirm these findings in a 


\section{Table 2: An Overview of the Existing Nerve Ultrasound Studies on Chronic Inflammatory Demyelinating Polyneuropathy and their Pathologic Findings}

\begin{tabular}{|c|c|c|c|c|c|c|c|c|c|}
\hline Author/s & $\begin{array}{l}\text { Patients } \\
\text { (n) }\end{array}$ & $\begin{array}{l}\text { Controls } \\
\text { (n) }\end{array}$ & $\begin{array}{l}\text { Median } \\
\text { Nerve }\end{array}$ & $\begin{array}{l}\text { Ulnar } \\
\text { Nerve }\end{array}$ & $\begin{array}{l}\text { Brachial, Plexus, } \\
\text { or Cervical Roots }\end{array}$ & Sciatic Nerve & Femoral Nerve & Fibular Nerve & Tibial Nerve \\
\hline Taniguchi et al..$^{30}$ & 1 & - & $x$ & $x$ & - & $x$ & $x$ & - & - \\
\hline Matsuoka et al. ${ }^{32}$ & 13 & 35 & - & - & $x$ & - & - & - & - \\
\hline Imamura et al. ${ }^{33}$ & 1 & - & $x$ & - & - & - & - & - & - \\
\hline Zaidman et al. ${ }^{15}$ & 36 & 90 & $x$ & $x$ & - & - & - & - & - \\
\hline Kerasnoudis et al. ${ }^{19}$ & 4 & 30 & $x$ & $x$ & - & - & - & $\mathrm{N}$ & - \\
\hline Kerasnoudis et al. ${ }^{21}$ & 1 & 30 & $x$ & $x$ & $x$ & - & - & $\mathrm{N}$ & $\mathrm{N}$ \\
\hline Jang et al. ${ }^{34}$ & 1 & - & $x$ & $x$ & - & - & - & $x$ & $x$ \\
\hline
\end{tabular}

$x=$ the concrete nerve was reported with pathologic findings; $N=$ the concrete nerve was reported with normal findings; $-=$ the concrete nerve was not studied at all; $n=$ absolute number.

later study. ${ }^{15}$ Systematic studies on the sensitivity and specificity of this finding failed in the literature. A novel approach to the quantification of the pathologic findings in CIDP was recently published in the literature. ${ }^{28,29}$ Using two new measures, the intranerve and internerve CSA variability, in a small group of immune-mediated neuropathies, Padua et al. and Kerasnoudis et al. were able to demonstrate that the CIDP favourable shows a diffuse pattern of nerve enlargement (lower values of intranerve CSA variability) compared with other immune-neuropathies, such as the multifocal motor neuropathy (MMN) (higher values of intranerve CSA variability).

Two reported CIDP cases aimed to highlight the possible value of neuromuscular ultrasound as a screening tool of immune therapy. In both cases, following intravenous immunoglobulin or prednisolone therapy, the patients experienced a remarkable clinical improvement, but the sonographic follow up did not show any improvement of the pathologic findings. ${ }^{33,35}$ The same observation was made in three cases of multifocal acquired demyelinating sensory and motor neuropathy (MADSAM). ${ }^{20,21}$ Systematic data on the sensitivity and specificity of sonography as a screening method of immune therapy do not exist.

\section{Conclusions}

As the main uncertainties regarding the diagnostic criteria of CIDP are steadily resolved, new challenges continuously arise on how to acquire the best static and dynamic imaging of the relevant nerve structures in this type of immune-mediated disease, aiming to provide a complementary and holistic approach to nerve impairment. Although the first nerve ultrasound studies on CIDP are rewarding to both clinicians and patients, the challenge remains to quantify ultrasound changes and to highlight a possible unique distribution pattern of pathologic findings. The newly proposed measurements in the literature ${ }^{28,29}$ may help to achieve this goal, but multicenter prospective validation and clinical correlations are required.
1. McLeod JG, Pollard JD, Macaskill P, et al., Prevalence of chronic inflammatory demyelinating polyneuropathy in New South Wales Australia, Ann Neurol, 1999;46:910-13.

2. Lunn MP, Manji H, Choudhary PP, et al., Chronic inflammatory demyelinating polyradiculoneuropathy: a prevalence study in south east England, J Neurol Neurosurg Psychiatry, 1999;66:677-80.

Dalakas MC, Advances in the diagnosis, pathogenesis and treatment of CIDP, Nat Rev Neurol, 2011;7:507-17.

Lewis RA, Chronic inflammatory demyelinating polyneuropathy, Neurol Clin, 2007;25:71-87.

5. Koller $\mathrm{H}$, Kieseier $\mathrm{BC}$, Jander S, Hartung HP, Chronic inflammatory demyelinating polyneuropathy, N Eng/ J Med, 2005;352:1343-56.

Rajabally YA, Nicolas G, Pieret F, et al., Validity of diagnostic criteria for chronic inflammatory demyelinating polyneuropathy: a multicen European study, J Neurol Neurosurg Psychiatry, 2009;80:1364-8. Saperstein DS, Chronic acquired demyelinating polyneuropathies, Semin Neurol, 2008;28:168-84

8. Cornblath DR, Feasby TE, Hahn AF, et al., Research criteria for diagnosis of chronic inflammatory demyelinating polyneuropathy (CIDP), Neurology, 1991:41:617-18.

9. Midroni G, de Tilly LN, Gray B, Vajsar J, MRI of the cauda equina in CIDP clinical correlations, I Neurol SCi, 1999;170:36-44.

Duggins AJ, McLeod JG, Pollard JD, et al., Spinal root and plexus hypertrophy in chronic inflammatory demyelinating polyneuropathy, Brain, 1999; 122:1383-90.

11. Eurelings $\mathrm{M}$ Notermans $\mathrm{NC}$, Franssen $\mathrm{H}$, et al., MRI of the brachial plexus in polyneuropathy associated with monoclonal gammopathy, Muscle Nerve, 2001;24:1312-18.

12. Sinclair CD, Morrow JM, Miranda MA, et al., Skeletal muscle MRI magnetisation transfer ratio reflects clinical severity in periphera neuropathies, J Neurol Neurosurg Psychiatry, 2012;83:29-32.

13. Mizuno K, Nagamatsu M, Hattori N, et al., Chronic inflammatory demyelinating polyradiculoneuropathy with diffuse and massive peripheral nerve hypertrophy: distinctive clinical and magnetic resonance imaging features, Muscle Nerve, 1998,21:805-8.
Schady W, Goulding PJ, Lecky BR, et al., Massive nerve roo enlargement in chronic inflammatory demyelinating polyneuropathy,
ent
I Neurol Neurosurg Psychiatry, 1996;61:636-40.

15. Zaidman CM, Al-Lozi M, Pestronk A, Peripheral nerve size in norma and patients with polyneuropathy: an ultrasound study, Muscle Nerve 2009:40:960-66

16. Smith EC, Hobson-Webb LD, Massey E, Nerve ultrasound in motor conduction b lock: pre- and posttreatment findings, Muscle Nerve 2008:38:1369

17. Granata G, Pazzaglia C, Calandro P, et al., Ultrasound visualization of nerve morphological alteration at the site of conduction block, Muscle Nerve, 2009;40:1068-70

18. Beekman R, van den Berg $\mathrm{LH}$, Franssen $\mathrm{H}$, et al., Ultrasonography shows extensive nerve enlargements in multifocal motor neuropathy,

19. Kerasnoudis A, Correlation of sonographic and electrophysiological findings in a patient with multifocal motor neuropathy, I Neuroimaging,

20. Scheidl E, Böhm J, Simó M, et al., Ultrasonography of MADSAM neuropathy: focal nerve enlargements at sites of existing and resolved

condis

1. Kerasnoudis A, Ultrasonography of MADSAM neuropathy: foca nerve enInd resolved conduction blocks, Neuromuscul Disord,

22. Cartwright MS, Passmore LV, Yoon JS, et al., Cross-sectional area reference values for nerve ultrasonography, Muscle Nerve

. Cartwright MS, Shin HW, Passmore LV, Walker FO, Ultrasonographic Jerence values for assessing the normal median nerve in adults,

24. Cartwright MS, Shin HW, Passmore LV, Walker FO, Ultrasonographic findings of the normal ulnar nerve in adults, Arch Phys Med Rehabil, 2007;88:394-6

25. Yalcin $\mathrm{E}$, Onder $\mathrm{B}$, Akyuz $\mathrm{M}$, Ulnar nerve measurements in healthy individuals to obtain reference values, Rheumatol Int, 2013;33(5):1143-7.

26. Won SJ, Kim BJ, Park KS, et al., Measurement of cross-sectional area of cervical roots and brachial plexus trunks, Muscle Nerve,

46:711-16.

27. Haun DW, Cho JC, Kettner NW, Normative cross-sectional area of the C5-C8 nerve roots using ultrasonography, Ultrasound Med Biol, 2010;36:1422-30.

28. Padua L, Martinoli C, Pazzaglia C, et al., Intra- and internerve crosssectional area variability: new ultrasound measures, Muscle Nerve, 2012; $45: 730-3$.

29. Kerasnoudis A, Klasing A, Behrendt V, et al., Intra- and internerve crosssectional area variability: new ultrasound measures, Muscle Nerve, 2013:47:146-7.

30. Taniguchi $\mathrm{N}$, Itoh $\mathrm{K}$, Wang $\mathrm{Y}$, et al., Sonographic detection of diffuse peripheral nerve hypertrophy in chronic inflammatory demyelinating polyneuropathy, elin Ultrasound, 2000;28:488-91.

31. Matsuda M, Ikeda S, Sakurai S, et al., Hypertrophic neuritis due to chronic inflammatory demyelinating polyradiculoneuropathy (CIDP): a postmortem pathological study, Muscle Nerve, 1996;19:163-9,

32. Matsuoka N, Kohriyama T, Ochi K et al., Detection of cervical nerve root hypertrophy by ultrasonography in chronic inflammatory demyelinating

33. Imamura K, Tajiri Y, Kowa H, Nakashima K, Peripheral nerve hypertrophy in chronic inflammatory demyelinating polyradiculoneuropathy detected by ultrasonography, Intern Med, 2009;48:581-2.

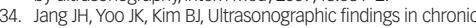
inflammatory demyelinating polyneuropathy, Am J Phys Med Rehabil, 2012;91:546-7

35. Kerasnoudis $A$, Nerve ultrasound in a case of chronic inflammatory demyelinating neuropathy, Muscle Nerve, 2013;47(3):443-6.

36. demyelinating polyneuropathy, Am J Phys Med Rehabil, 2013;39(6):1129. . Kerasnoudis A, Gold R, Klasing A, et al., Correlation of morphological changes in CIDP with the disease duration. DGN Congress. 85 . Kongress der Deutschen Geselschaft für Neurologie (DGN), 27.09.12, Hamburg Germany. P555.

38. Rajabally YA, Morlese J, Kathuria D, Khan A,Median nerve ultrasonography in distinguishing neuropathy sub-types: a pilot study, Acta Neurologica Scandinavica, 2012;125:254-9.

39. Smith EC, Hobson-Webb LD, Massey E, Nerve ultrasound in motor conduction block: pre- and posttreatment findings, Muscle Nerve,
2008:38:1369. 